\title{
La población docente de Informática Educativa en Costa Rica: sus conocimientos, disposiciones y prácticas habituales
}

\author{
$\ldots \ldots \ldots \ldots \ldots \ldots \ldots \ldots \ldots \ldots \ldots \ldots \ldots \ldots \ldots \ldots \ldots \ldots \ldots \ldots \ldots \ldots \ldots \ldots \ldots \ldots \ldots \ldots \ldots$ \\ Katherine Vindas Sánchez ${ }^{1} \&$ Melania Brenes Monge ${ }^{2}$ \\ 1. Investigadora, Fundación Omar Dengo, Costa Rica; katherine.vindas@fod.ac.cr \\ 2. Coordinadora de Investigación y Evaluación, Fundación Omar Dengo, Costa Rica; melania.brenes@fod.ac.cr
}

Recibido: 09 de noviembre del 2016

Corregido: 06 de diciembre del 2016

Aceptado: 23 de enero del 2017

\begin{abstract}
Resumen
El presente estudio explora las características del personal docente costarricense que atiende primaria y secundaria en la disciplina de Informática Educativa, en términos de sus conocimientos, disposiciones y prácticas en el quehacer profesional. Los hallazgos permitieron identificar cuatro perfiles diferentes en esta población en dos dimensiones principales: práctica pedagógica y desarrollo profesional docente. Además, se expone que la población de docentes de Informática Educativa tiene acceso prácticamente generalizado a las tecnologías y hacen usos sofisticados de esta, primordialmente en el ámbito personal. La mayoría cuenta con un perfil que revela una implementación de la propuesta educativa de acuerdo con lo esperado y con una disposición favorable hacia el desarrollo profesional.
\end{abstract}

Palabras clave: Tecnologías de la información, tecnología educacional, informática educativa, eficacia del docente, mejora de la educación.

\begin{abstract}
Educational Informatics teachers in Costa Rica: their knowledge, attitudes and habitual practices

This study explores the current state of the Costa Rican Educational Informatics Teachers' performance in terms of their knowledge, attitudes and habitual practices in their professional work. The findings allowed to identify four different profiles in two main dimensions: pedagogical practice and teacher professional development. These results point to say that Educational Informatics Educators are a population with virtually universal access to technologies and sophisticated applications especially in their personal sphere. The majority of them are in the profiles that revels an implementation of the educational proposal as expected, and with a favourable disposition towards professional development.
\end{abstract}

Key words: Information technologies, educational technologies, educational informatics, teachers' efficiency, educational improvement. 


\section{INTRODUCCIÓN}

Gracias al trabajo articulado de la Fundación Omar Dengo y el Ministerio de Educación Pública, el Programa Nacional de Informática Educativa MEP - FOD (PRONIE MEP - FOD) es una de las iniciativas más amplias y permanentes en el Sistema Educativo Público Costarricense. El PRONIE MEP - FOD en sus diversas propuestas de aprovechamiento educativo de las tecnologías digitales se orienta hacia objetivos de desarrollo de capacidades en las personas para actuar como protagonistas en la sociedad actual. Según Muñoz et. al (2014) dos planteamientos han orientado las propuestas, una visión vanguardista de que el aporte de las tecnologías en la educación está centrado en el desarrollo de capacidades estratégicas y una clara idea de que el acceso a las tecnologías por sí mismas no genera ningún resultado significativo sin una propuesta educativa asociada.

En el año 1992, como parte de la trayectoria e hitos históricos más relevantes del PRONIE MEP - FOD, en convenio con la Universidad Estatal a Distancia (UNED), se realiza la apertura de la carrera de Informática Educativa en grados de bachillerato y licenciatura. Y es así como comienza el desarrollo de esta especialidad docente en el país. A diciembre del 2015, el MEP tiene nombrados a aproximadamente 1399 docentes en todo el país, trabajando en los laboratorios de Informática Educativa del Programa.

EI PRONIE MEP - FOD ha realizado esfuerzos permanentes de acompañar sus procesos educativos con un trabajo continuo de investigación y evaluación formativa para desarrollar enfoques, estrategias y metodologías capaces de formar al profesorado para aproximarse a la complejidad de resultados generados por el Programa. En este contexto, la investigación permanente sobre el perfil de la población de Informática Educativa con la que trabaja ha sido muy importante. Así, en el año 2015, se plantea la necesidad de conocer las características del personal docente de Informática Educativa de primaria y secundaria en términos de conocimientos, disposiciones y prácticas que realizan en su quehacer profesional.

Los resultados de este estudio en el contexto del PRONIE MEP - FOD, orientan las labores descritas brindando información de la realidad del profesorado, el establecimiento de una línea base de sus perfiles $y$, por tanto, brindan la posibilidad de hacer propuestas de desarrollo profesional adaptadas a las necesidades reales de esta población.

La investigación efectuada tuvo como objetivo general identificar los conocimientos, las disposiciones y las prácticas habituales del sector docente en Informática Educativa para orientar la oferta de desarrollo profesional y la actualización de la propuesta pedagógica de los estudiantes. Para alcanzar este objetivo, se planteó:

1. Describir las prácticas, conocimientos y disposiciones del personal docente asociados a la implementación de las propuestas pedagógicas.

2. Caracterizar las prácticas, conocimientos y disposiciones del personal docente en relación con sus propios procesos de desarrollo profesional

En la dimensión de práctica pedagógica, se entiende el aprovechamiento de las tecnologías por parte del sector docente:

En función del diseño, mediación y evaluación de procesos de enseñanza-aprendizaje, en los que se modela para los estudiantes los usos contemporáneos, seguros y responsables de las tecnologías (...) para alcanzar los resultados de aprendizaje esperados con mayor calidad y eficiencia. (Fundación Omar Dengo, en prensa, p.20). 
En la dimensión de desarrollo profesional:

La gestión y liderazgo de la propia formación continua, en colaboración con los pares. Se pretende que como miembro activo de una comunidad profesional, los docentes aprovechen el potencial de las tecnologías, especialmente de Internet, para participar en entornos virtuales, mantenerse actualizados, reflexionar y construir conocimiento. (Fundación Omar Dengo, en prensa, p.21)

Dentro de los hallazgos más relevantes del estudio se señala la identificación de cuatro perfiles de docentes de Informática Educativa asociados a estas dimensiones. Estos perfiles son los siguientes:

1. Poco dispuestos a actualizarse y rezagados en la ejecución de la propuesta

2. Dispuestos a actualizarse pero poco conocedores de la propuesta

3. Poco dispuestos a actualizarse pero conocedores de la propuesta

4. Dispuestos a actualizarse y conocedores de la propuesta

El presente trabajo describe las principales características de las personas docentes de Informática Educativa participantes a nivel general, y el detalle de sus características según las dimensiones y los perfiles obtenidos.

Las autoras agradecen a directores y docentes de Informática Educativa de los centros educativos participantes por su colaboración en el estudio. Asimismo, el trabajo se desprende de un estudio realizado por un equipo de investigadores de la Unidad de Evaluación de la Fundación Omar Dengo, bajo la dirección de Magaly Zúñiga Céspedes, a quiénes se les reconoce su compromiso y aporte en vías del fortalecimiento de las propuestas educativas apoyadas con tecnologías digitales en Costa Rica.

\section{METODOLOGÍA}

El estudio desarrollado corresponde a una investigación cuantitativa de tipo descriptivo que utiliza análisis multivariado por conglomerados. En esta investigación participaron 873 docentes de Informática Educativa de los 1390 registrados a nivel nacional. De esta muestra, 726 eran docentes de primaria y 147 de secundaria.

El promedio de edad de las personas participantes fue de 38 años y la mayoría se ubicó en el rango de los 30 a 39 años. En cuanto a la distribución por sexo, de los 726 docentes de primaria 516 eran mujeres, y en secundaria, de los 147 docentes 90 eran mujeres. El requisito para participar del estudio era contar con conexión a Internet en alguno de los centros educativos donde se desempeña para completar un instrumento en línea.

Al momento del estudio, en octubre del 2015, el Programa beneficiaba a 1158 centros educativos. Esta cantidad puede incrementar de un año a otro por el aumento de cobertura que procura el Programa. En total se recolectó información para el $65,6 \%$ de los centros educativos y el 62,8\% del profesorado que imparte la propuesta. El tamaño de muestra obtenido permite lograr una valoración de la situación actual de la población docente de manera generalizada y pertinente para los propósitos del presente estudio. 
TABLA 1

Centros educativos, docentes y porcentaje de cobertura del estudio según población meta

\begin{tabular}{|c|c|c|c|}
\hline \multirow{2}{*}{ Descripción } & \multicolumn{2}{|c|}{ Nivel } & \multirow{2}{*}{ Total } \\
\hline & Primaria & Secundaria & \\
\hline \multicolumn{4}{|c|}{ Centros Educativos } \\
\hline Población & 935 & 223 & 1158 \\
\hline Consultados & 636 & 124 & 760 \\
\hline Cobertura (\%) & $68,0 \%$ & $55,6 \%$ & $65,6 \%$ \\
\hline \multicolumn{4}{|c|}{ Docentes } \\
\hline Población & 1056 & 334 & 1390 \\
\hline Consultados & 726 & 147 & 873 \\
\hline Cobertura (\%) & $68,8 \%$ & $44,0 \%$ & $62,8 \%$ \\
\hline
\end{tabular}

Fuente: Fundación Omar Dengo, 2016, Estudio de conocimientos, prácticas y disposiciones de los docentes de Informática Educativa 2015, p. 5.

Para la recolección de información, se elaboró un cuestionario digital de autoreporte creado en la plataforma de Google Spreed Sheets. Este cuestionario está organizado en tres apartados: 1. información general, 2. desarrollo profesional y 3. práctica pedagógica. En la sección de información general, se indagó acerca de datos personales y sociodemográficos, formación académica y experiencia. Sobre las restantes dimensiones, se investigaron los conocimientos, las prácticas y disposiciones correspondientes. El instrumento está constituido por 98 ítems de distintos tipos: selección única, selección múltiple, escalas y preguntas abiertas.

El proceso de investigación se desarrolló por medio de tres etapas que se describen a continuación. En la primera etapa, se realizó una investigación documental sobre evaluación de docentes en temas de educación y aprovechamiento de tecnología en el quehacer docente. En la segunda etapa, se definieron las categorías de análisis y variables para responder a los objetivos. Se seleccionaron ítems y escalas de medición validadas en estudios anteriores de la Unidad de Investigación y Evaluación de la Fundación Omar Dengo para el desempeño docente. Adicionalmente, se crearon una serie de ítems para completar los requerimientos evaluativos del estudio. En esta etapa, se generó la primera versión del cuestionario. Posterior a esto, el cuestionario se validó y depuro con asesores de laboratorios de Informática Educativa (LIE) del PRONIE MEP-FOD y mediante la aplicación de ocho entrevistas cognitivas a docentes de Informática Educativa, de primaria (4 participantes), secundaria y de zonas tanto urbana como rural (4 participantes).

Por último, en la tercera etapa, se digitalizó el cuestionario y se generó el vínculo de acceso para el profesorado. Éste fue enviado por correo electrónico a los centros educativos que cuentan con laboratorio de Informática Educativa y con acceso a Internet para ser completado. El cuestionario podía ser llenado una única vez por quienes participaban en el estudio.

En el análisis de datos, se emplearon técnicas de estadística descriptiva e inferencial, útiles para describir la población y explorar posibles relaciones o influencia de variables de tipo sociodemográfico y de información general con la información de desarrollo profesional y práctica pedagógica.

Para describir al sector docente en las dimensiones de práctica pedagógica y de desarrollo profesional se consideraron características deseables establecidas en las competencias docentes, así como una serie 


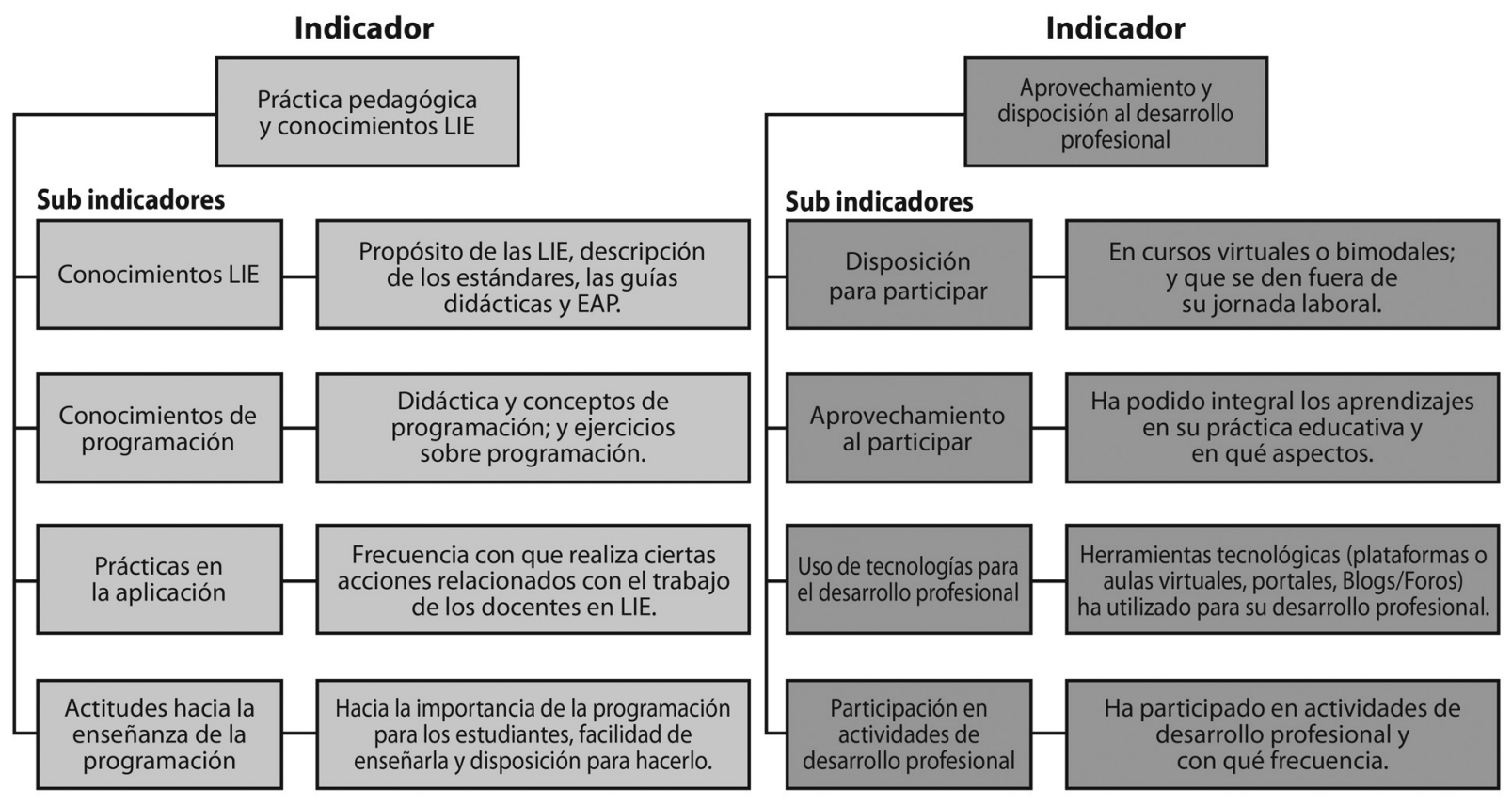

Figura 1. Sub indicadores y componentes de los indicadores de Aprovechamiento y disposición al desarrollo profesional, práctica pedagógica y conocimientos LIE. Fuente: Fundación Omar Dengo, 2016, Estudio de conocimientos, prácticas y disposiciones de los docentes de Informática Educativa 2015, p. 7.

de conocimientos, actitudes y prácticas que se espera que el personal docente realice en la actualidad como parte de su rol profesional y como docente de Informática Educativa según el PRONIE MEP - FOD.

Para este análisis, se crearon indicadores y subindicadores de conocimientos, prácticas y disposiciones de cada dimensión (ver Figura 1). La puntuación de estos indicadores se estableció en un gradiente de 0 a 10. Los mayores puntajes correspondían a las respuestas donde se reportaba mayor disposición, actitudes positivas, prácticas deseables en su trabajo habitual y los conocimientos requeridos para un adecuado desempeño

Se utilizó análisis multivariado mediante la técnica de conglomerados para distinguir perfiles de docentes y lograr establecer comparaciones entre ellos. Para esto, se tomaron como referencia las puntuaciones en los indicadores de las dos dimensiones evaluadas.

\section{RESULTADOS}

\section{Generalidades sobre la población docente de Informática Educativa}

En términos de la experiencia docente, las personas docentes poseen un promedio similar de años de experiencia en Informática Educativa. El profesorado de primaria reportó un promedio de 10 años; el de secundaria, de 12 años. Para la mayoría de educadores de primaria su experiencia principal es en esta especialidad. En cambio, el personal docente de secundaria es un poco más experimentado en otras disciplinas diferentes a la Informática Educativa. 
En cuanto a la formación académica, la mayoría cuenta con licenciatura, $63 \%$ en primaria y $61 \%$ en secundaria. Los otros grados académicos que poseen son bachillerato, maestría, especialidad, profesorado y diplomado respectivamente. Actualmente, $23 \%$ de quienes participaron en el estudio se encuentran aun estudiando.

El acceso a tecnología en esta población es muy generalizado, $90 \%$ de quienes participaron reportaron tener computadoras y teléfonos inteligentes; y $83 \%$ posee conexión permanente a Internet desde su teléfono.

En relación con el uso de estos dispositivos, el uso del celular es generalizado y diario. Esto se perfila diferente al uso de su computadora, donde es $49 \%$ del profesorado el que la usa diariamente. Existen diferencias significativas en cuanto a mejores disposiciones de los docentes de primaria para usar el teléfono para "revisar información relativa a su profesión" y "participar en grupos de WhatsApp relacionados con su trabajo".

Al respecto del acceso a Internet en el hogar, el dispositivo que más usan es la computadora. Así 82\% del personal docente accede por este medio. Por el contrario, el celular es el recurso menos utilizado para este fin, solamente por $23 \%$ de las personas participantes. En el centro educativo, la computadora del LIE es el dispositivo mayormente empleado para acceder a este servicio, reportado por $84 \%$ de quienes participaron en el estudio. Mientras que la tableta todavía no representa un dispositivo de uso cotidiano para trabajar y conectarse a Internet.

El sector docente que participó en el estudio reporta capacitarse principalmente en temas relacionados con la propuesta educativa tal como la programación, y en menor medida con temas vinculados al uso de las potencialidades de Internet como redes sociales o instalación de redes. También, se consultó acerca de capacitaciones en temas como: uso de internet, redes sociales, ofimática, multimedia y uso de las tecnologías para enseñar, resolución de problemas de programación, programación y didáctica de la programación. En lo relacionado con su nivel de dominio en estos temas reportaron en su mayoría poseer un nivel intermedio en los temas relacionados con programación e intermedio-alto en el resto de temas.

Alrededor de 55\% de docentes indica tener limitaciones para utilizar herramientas tecnológicas en su desarrollo profesional, siendo las principales razones la falta de oportunidades (22\%), el desconocimiento (18\%) y el uso solo a nivel personal (14\%). Otras razones más específicas que reportan están relacionadas con el tipo de herramienta tecnológica. Indican que los chats pueden ser poco útiles o inapropiados para el desarrollo profesional y que la participación en blog/foros, redes sociales, comunidades virtuales, aulas virtuales y sitios web no se utiliza con estos fines porque se les otorga un uso más personal o por la falta de tiempo y de conexión a Internet.

En términos de los niveles de uso de la tecnología, se identificaron dos dimensiones: la primera de "nociones básicas" que se refiere al uso para almacenar información en un dispositivo, editar texto, compartir fotografías y videos e imprimir documentos. La segunda de "profundización y generación del conocimiento" que hace referencia al manejo de hojas de cálculo, utilizar un lenguaje de programación, uso de software libre, elaborar y participar en blogs, usar software para la elaboración de recursos didácticos y construcción de mapas conceptuales, participar en cursos virtuales, usar sitios web para acceder o cancelar servicios y participar en redes sociales para compartir experiencias. Ambas dimensiones de uso de la tecnología se aproximaron desde dos ámbitos de aplicación: el uso para la enseñanza y el uso personal tal como se muestra en la Figura 2. Para el análisis de estas dimensiones, se creó un indicador para cada dimensión en una escala de 1 a 10, en donde 10 es el valor más alto.

El nivel de uso de las tecnologías digitales es mayor en el ámbito personal que en el ámbito educativo, y la dimensión de "nociones básicas" presenta puntajes más altos en ambos casos. Lo anterior parece 


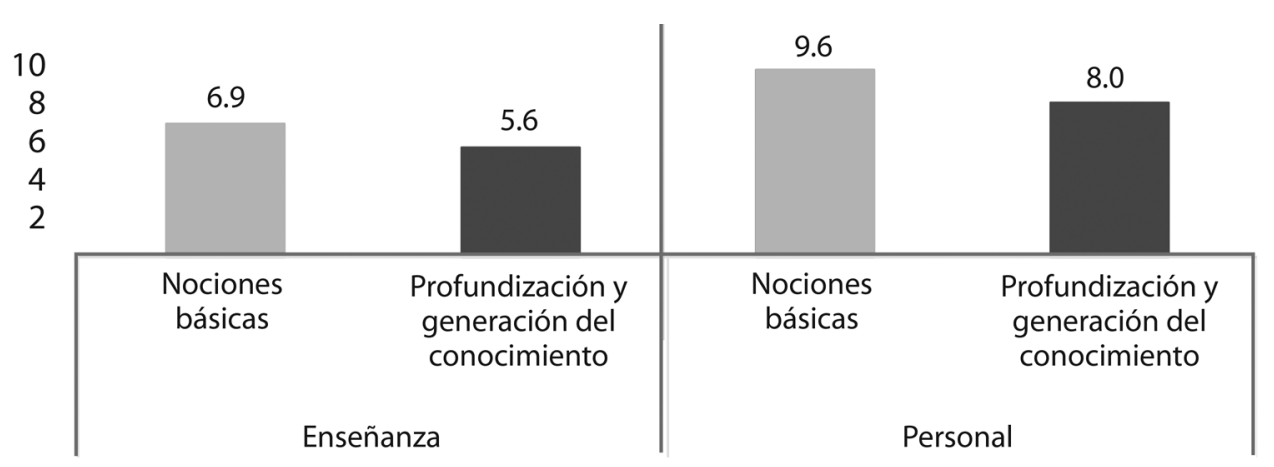

Figura 2. Nivel de uso de tecnologías para la enseñanza y para uso personal. Fuente: Fundación Omar Dengo, 2016, Estudio de conocimientos, prácticas y disposiciones de los docentes de Informática Educativa 2015, p. 18

evidenciar que el personal docente tiene conocimiento y utiliza los recursos tecnológicos en niveles altos para su beneficio personal, pero no los están implementando en la enseñanza.

\section{Perfiles identificados en los docentes de Informática Educativa}

Como parte del análisis de conglomerados se buscó definir perfiles para clasificar al sector docente de Informática Educativa según sus características relacionadas con el desarrollo profesional y su práctica pedagógica. Los perfiles son los siguientes:

\section{A. Poco dispuestos a actualizarse y rezagados en la ejecución de la propuesta}

B. Dispuestos a actualizarse, pero poco conocedores de la propuesta

\section{Poco dispuestos a actualizarse, pero conocedores de la propuesta}

\section{Dispuestos a actualizarse y conocedores de la propuesta}

Figura 3. Distribución de docentes según conglomerados resultantes de las características en práctica pedagógica y desarrollo profesional. Fuente: Fundación Omar Dengo, 2016, Estudio de conocimientos, prácticas y disposiciones de los docentes de Informática Educativa 2015, p. 25

A continuación, un análisis detallado de los resultados obtenidos en los perfiles identificados.

\section{Perfil A. Poco dispuestos a actualizarse y rezagados en la ejecución de la propuesta}

Este perfil está constituido por 111 docentes, 12,7\% de la muestra. Este grupo de docentes atiende principalmente población de secundaria, tiene pocos años de experiencia (menos de 4 años) y no ha concluido grados académicos superiores. El grupo evidencia resistencia hacia el desarrollo profesional en general. Casi no han participado de oportunidades de este tipo. Prefieren, cursos presenciales y argumentan desconocimiento, falta de tiempo, de oportunidades y de acceso a Internet para no utilizar 
herramientas tecnológicas para su desarrollo profesional. Evidencian una implementación de la propuesta educativa insuficiente debido a los pocos conocimientos que poseen sobre los contenidos y los objetivos primordiales de esta.

Al indagar sobre la actividad de programación en los LIE, este perfil de docente opina que a los estudiantes no les interesa esta área, aunque reconocen que es útil para su futuro, sus consideraciones se enfocan más en las dificultades que enfrentan al momento de enseñar a programar (tiempo, dificultad de la propuesta, etcétera).

El nivel de uso de la tecnología de este grupo de docentes en el ámbito personal es similar al de los otros perfiles, es decir por encima de la media. No obstante, su nivel de uso de la tecnología para enseñar es bajo comparado con los otros perfiles. Predomina el uso personal de la tecnología sin articulación con la labor docente.

\section{Perfil B. Dispuestos a actualizarse pero poco conocedores de la propuesta}

El perfil está representado por 263 docentes, 30,1\% de la muestra. El grupo, principalmente constituido por población de secundaria, posee una cantidad considerable de años de experiencia (más de 10), y han alcanzado los grados académicos superiores (licenciatura, maestría). Evidencian disposición favorable hacia el desarrollo profesional, así como a participar de oportunidades de este tipo tanto presenciales como virtuales.

El perfil en particular está conformado por personas docentes que muestran pocos conocimientos sobre los contenidos y objetivos de la propuesta educativa que imparten, por lo que su implementación no es la esperada. Además, consideran que el estudiantado no está interesado en aprender a programar, pero son conscientes de la utilidad de tener competencias en esta área para el futuro y para el desarrollo de sus estructuras de pensamiento. Cuando hacen referencia a este ámbito, se centran en las dificultades que representa para la población estudiantil, pero también reconocen la dificultad que involucra enseñarlo.

El uso de la tecnología que muestran en lo personal es similar al de los otros perfiles, por encima de la media. Mientras que su nivel de uso de la tecnología para enseñar, al igual que otros perfiles, está por debajo de la media. En este perfil sigue existiendo una brecha entre usar la tecnología en el ámbito personal y el académico.

\section{Perfil C: Poco dispuestos a actualizarse pero conocedores de la propuesta}

Este perfil está representado por 190 docentes, 21,8\% de la muestra. Grupo conformado por población docente de secundaria principalmente, tiene una cantidad de años de experiencia considerable (menos de 9 años de experiencia), pero no ha concluido grados académicos superiores.

Se caracterizan por resistencia hacia el desarrollo profesional en general. Se capacitarían solamente de forma presencial. Entre las razones que dan para no usar herramientas tecnológicas en su desarrollo profesional se hallan la falta de oportunidades, el tiempo y el desconocimiento.

Con respecto a la implementación de la propuesta educativa, se califica como cercana a lo esperado pues evidencian conocimientos sobre los objetivos esperados e indican orientarse por el material de apoyo y las guías didácticas para la enseñanza.

Reconocen el valor de enseñar programación para el desarrollo de estructuras de pensamiento y para el futuro del estudiantado, aunque resaltan el desinterés que muestra esta población hacia aprender a programar. Destacan que para abordar correctamente el tema es importante la mediación docente y la introducción de mejoras a la propuesta del Programa. 
Su nivel de uso de la tecnología a nivel personal es similar al de los otros perfiles. Nuevamente, predominan los tipos de usos personales de la tecnología sin articulación con la labor docente.

\section{Perfil D. Dispuestos a actualizarse y conocedores de la propuesta}

Este perfil está conformado por un número más grande de docentes, 309 en total, 35,4\% de la muestra. El grupo está formado principalmente por población que atiende primaria, cuenta con más de 5 años de experiencia y ha obtenido grados académicos superiores.

Su disposición es favorable hacia el desarrollo profesional sea virtual o presencial e implementa la propuesta de forma cercana a lo esperado debido a los conocimientos que posee.

Así quienes conforman este grupo conocen los beneficios de enseñar programación en las lecciones de LIE, para el desarrollo de estructuras de pensamiento, el aprendizaje de estrategias para la resolución de problemas de la vida real y la utilidad para el futuro de la población estudiantil. Destacan la importancia de la mediación y el reto que representa para ellos como personal docente, pero esto no influye sus actitudes hacia enseñar a programar.

Su nivel de uso de la tecnología a nivel personal es similar al de los otros perfiles. No obstante, su nivel de uso de la tecnología para enseñar es el más alto frente a los otros perfiles. Asimismo, muestran los tipos de usos personales más altos, así como también los puntajes más altos en usos de recursos en línea y para la comunicación.

Los cuatro perfiles de docentes explorados muestran puntajes altos en el uso de la tecnología a nivel personal, esto quiere decir que muestran nociones básicas, de profundización y de generación de conocimiento con tecnologías. A nivel general el grupo A es el que obtiene los menores puntajes en los tipos de uso de la tecnología evaluados, los grupos B y C obtienen puntajes similares y el grupo D los puntajes más altos.

\section{Análisis de los perfiles en función de los indicadores y subindicadores de las dimensiones de práctica pedagógica y desarrollo profesional}

En términos del desarrollo profesional, la Figura 4 muestra los puntajes obtenidos en los subindicadores para cada uno de los perfiles de docentes.

El personal docente de Informática Educativa en general posee buena disposición para aprovechar oportunidades de crecimiento profesional y utilizar la tecnología con este propósito, especialmente aquellos que se encuentran en los perfiles $B$ y $D$. No obstante, existen diferencias entre los perfiles con respecto al aprovechamiento o la aplicación en el laboratorio de los conocimientos adquiridos en oportunidades de desarrollo profesional. El profesorado de estos perfiles podría tener baja tendencia para llevar a la práctica nuevas estrategias didácticas o nuevos conocimientos adquiridos en el trabajo con el estudiantado.

Por otra parte, en la dimensión de práctica pedagógica, se valoraron los conocimientos de la propuesta educativa de IE, los conocimientos sobre programación y las prácticas educativas en que aplican conocimientos adquiridos. Las Figuras 5 y 6 muestran los puntajes obtenidos por cada grupo de docentes en estos subindicadores.

En el gráfico anterior se puede destacar cómo las personas docentes de los cuatro perfiles obtienen las puntuaciones más bajas en relación con los conocimientos sobre programación; es decir alrededor de 


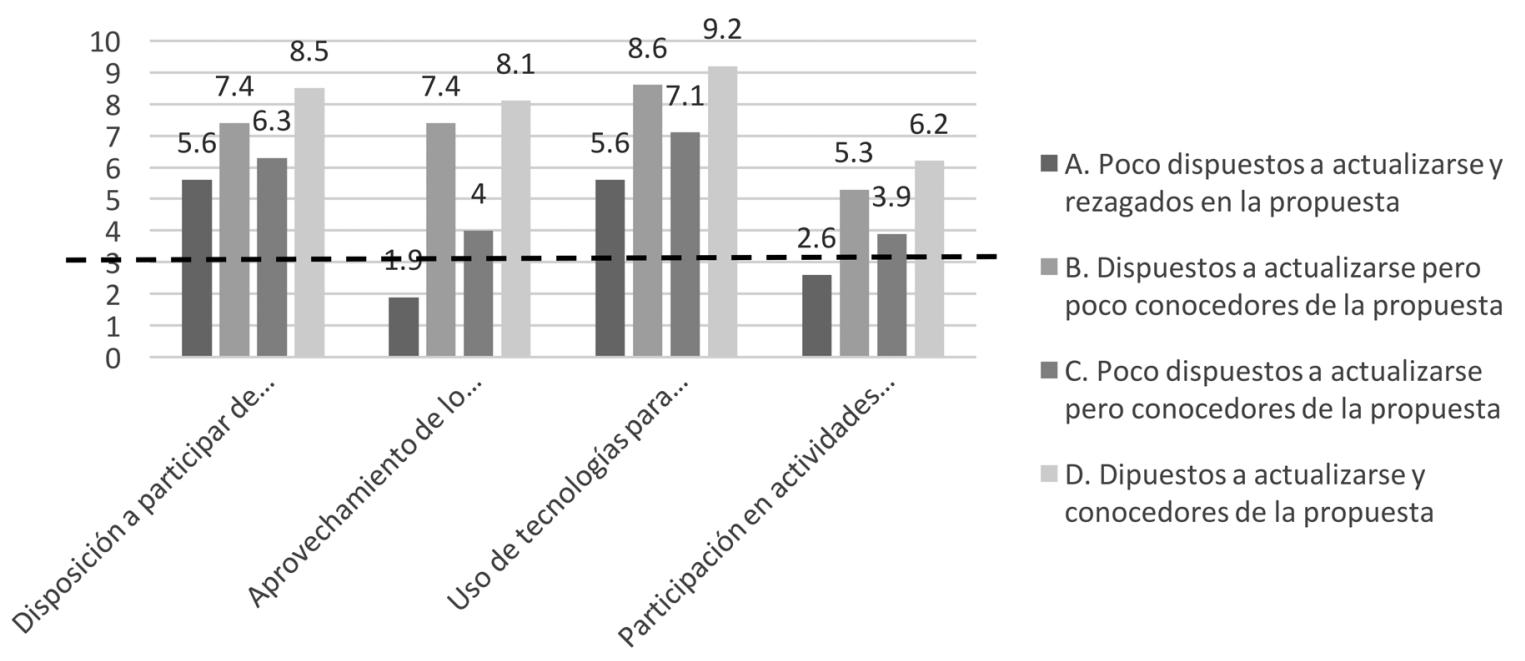

Figura 4. Puntajes promedio de indicadores de desarrollo profesional según conglomerados.

Fuente: Fundación Omar Dengo, 2016, Estudio de conocimientos, prácticas y disposiciones de los docentes de Informática Educativa 2015, p. 28.

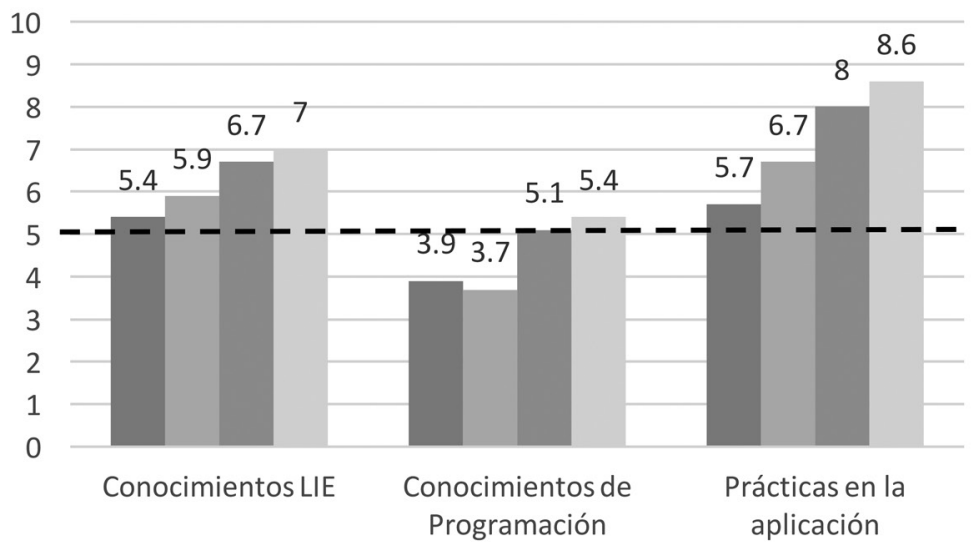

A. Poco dispuestos a actualizarse y rezagados en la propuesta

B. Dispuestos a actualizarse pero poco conocedores de la propuesta

- C. Poco dispuestos a actualizarse pero conocedores de la propuesta

D. Dipuestos a actualizarse y conocedores de la propuesta

Figura 5. Primera parte de puntajes obtenidos por los docentes de Informática Educativa en los subindicadores que componen el indicador de práctica pedagógica. Fuente: Fundación Omar Dengo, 2016, Estudio de conocimientos, prácticas y disposiciones de los docentes de Informática Educativa 2015, p. 30.

la media y con tendencia a la baja. Este resultado es muy relevante frente al diseño de una oferta de desarrollo profesional para educadores de esta especialidad. Al parecer, a pesar de la formación sostenida en esta área por parte del PRONIE MEP - FOD, sigue siendo un eje de mejora.

En los otros subindicadores las puntuaciones más altas son las relacionadas con las prácticas de docencia en las que se aplican en el aula conocimientos adquiridos. En cuanto a sus conocimientos de la propuesta de IE se colige que los cuatro perfiles indican un conocimiento mínimo, no obstante los perfiles C y D son los que presentan mejores puntajes en este subindicador.

Un hallazgo importante es que para la población de docentes el hecho de que el estudiantado aprenda a programar es valorado como positivo, a pesar de reconocer que existen dificultades para enseñarlo. 


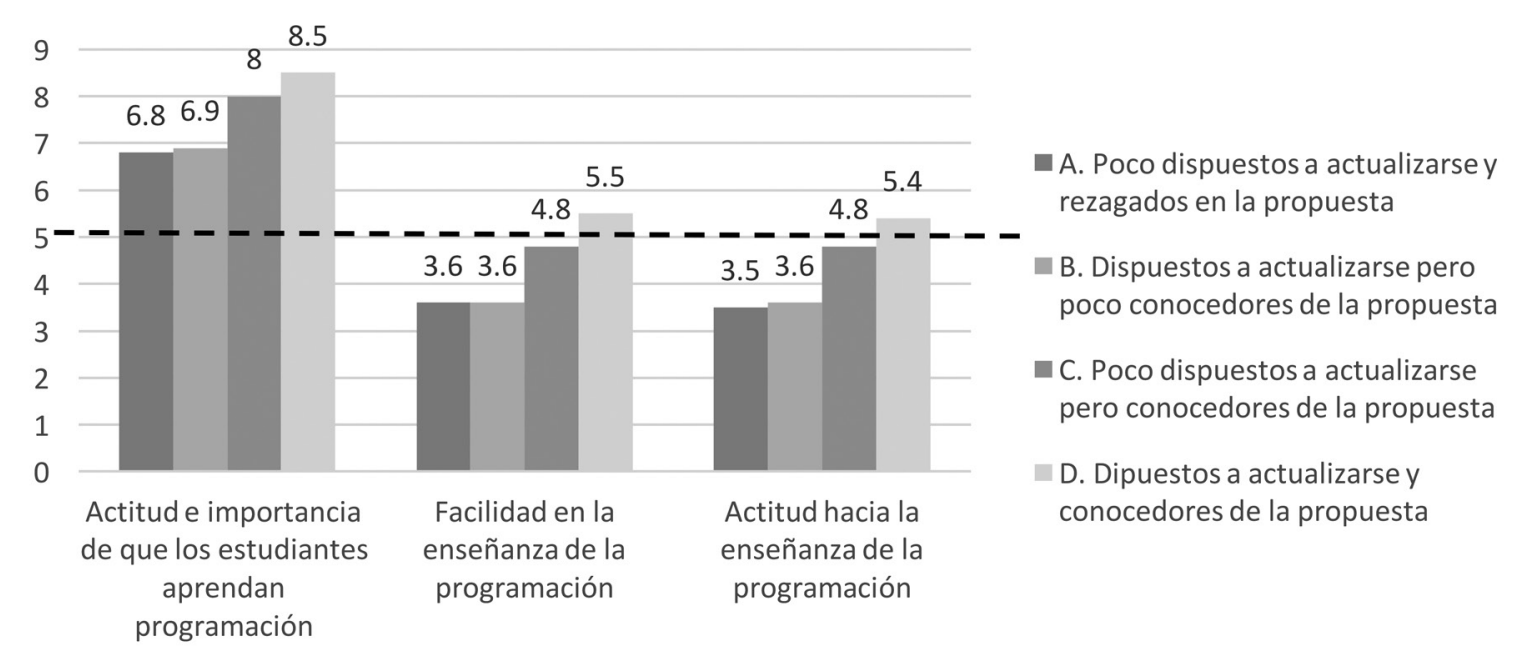

Figura 6. Segunda parte de puntajes obtenidos por los docentes de Informática Educativa en los subindicadores que componen el indicador de práctica pedagógica. Fuente: Fundación Omar Dengo, 2016, Estudio de conocimientos, prácticas y disposiciones de los docentes de Informática Educativa 2015, p. 31.

El estudio también exploró el concepto de programación que posee el personal docente de esta especialidad con el fin de identificar si existían conceptos más o menos sofisticados según cada perfil. No obstante, la mayoría de docentes, sin importar el perfil, evidenció conceptos cercanos y estrechamente relacionados con la acción de automatizar una solución o codificar. Únicamente existieron 18 contestaciones que hacían referencia a la relación entre el proceso de programar y el desarrollo de habilidades de pensamiento.

\section{DISCUSIÓN}

Los resultados obtenidos en esta investigación permiten caracterizar detalladamente la población de docentes de Informática Educativa desde sus fortalezas y oportunidades de mejora. La información es muy útil para desarrollar las ofertas formativas y el apoyo educativo necesario que les permita llevar a la práctica la propuesta educativa de mejor manera.

Se han identificado cuatro perfiles útiles para caracterizar la población de docentes de Informática Educativa desde su práctica pedagógica y desarrollo profesional. La mayoría del profesorado se encuentra en los perfiles con mejores puntajes en las dimensiones indagadas, también posee conocimientos adecuados que pone en práctica en su labor y algunas habilidades pedagógicas características de buenas prácticas en los laboratorios.

En cuanto a la experiencia como docentes de Informática Educativa, es la población que atiende primaria la que muestra menos cantidad de años laborando, pero la que todavía parece mantener mejores disposiciones para reflexionar sobre la realidad del contexto educativo, para capacitarse y para profundizar sus conocimientos sobre la propuesta educativa.

Los resultados anteriores son coherentes con las etapas de desarrollo profesional propuestas en el 2014 por el Instituto de Desarrollo Profesional Uladislao Gámez Solano del Ministerio de Educación Pública, las cuales se basan en los años de experiencia laboral. De esta forma, la población que atiende primaria se ubicaría en la etapa donde se demuestra mayor autonomía, búsqueda de actualización y consolidación 
de la práctica laboral (IDPUGS, 2014). De manera contraria, la mayoría de docentes de secundaria podrían estar pasando de la consolidación en el ejercicio de su profesión hasta una etapa de mayor criticidad sobre la trayectoria de su práctica laboral. Algunos de ellos incluso buscando cambios en los ámbitos donde se desenvuelven laboralmente (IDPUGS, 2014, p. 21).

Por otro lado, una de las características que llama la atención se relaciona con el desarrollo académico. La prevalencia de la licenciatura podría estar relacionada con el nivel académico en el cual la población docente encuentra mayor estabilidad laboral para nombramientos y categorías profesionales, por tanto no muestra la tendencia a seguir estudiando; en el caso de los bachilleres sí se observó que más de 50\% continúa con sus estudios.

Quienes participaron cuentan con acceso prácticamente generalizado a los recursos tecnológicos. Este hallazgo ya se ha reportado y respaldado anteriormente por otros trabajos como el de Lentini (2014) en su estudio de perfiles sociodemográficos de docentes costarricenses, y anteriormente por el estudio sobre la ruta de apropiación social de la tecnología de docentes costarricenses, realizado por Zúñiga, Núñez, Brenes y Chacón (2013).

En el caso del uso de las tecnologías por parte de la población docente nacional, el predominio del mayor provecho en el ámbito personal se ratifica con Zúñiga, Núñez, Brenes y Chacón (2013), quienes identifican una amplia brecha entre la complejidad del uso de las tecnologías en lo personal con respecto al uso en el aula. Este hallazgo apunta a que el proceso de apropiación tecnológica para la enseñanza por parte del profesorado parte de una primera etapa de aprovechamiento a nivel personal, y que se requieren condiciones e intervenciones educativas que permitan "interpretar" o articular el potencial de estos recursos en el proceso de enseñanza y aprendizaje para evolucionar y aprovechar los recursos en la dimensión educativa.

Muchos estudios sobre el uso de la tecnología en la educación han destacado la influencia de los conocimientos que debe tener el profesorado sobre el potencial de las tecnologías en la enseñanza para mejorar sus prácticas de integración en el aula. Lo anterior corresponde a lo identificado en este estudio. Es decir, quienes son conocedores de los aportes y objetivos del uso de la tecnología en función de resultados de aprendizaje en sus estudiantes muestran más y mejores disposiciones para generar usos diversificados y más profundos de la tecnología en el ámbito de la enseñanza. Así aunque el sector docente posea destrezas en el manejo de las tecnologías no es suficiente para aprovechar su potencial en los procesos de enseñanza y aprendizaje.

En cuanto a la participación en actividades de desarrollo profesional, si bien en su mayoría aplican los conocimientos adquiridos, todavía no parecen comprender que estas oportunidades les estén permitiendo realizar cambios o mejoras sustanciales en su práctica docente. Al respecto Salazar (2015) señala que:

El aprendizaje de los docentes de determinadas teorías, estrategias realizadas con los alumnos, se valida en el aula. Si dichas estrategias logran sus objetivos, resultados, se constata que el docente aprendió, y esta acción aporta al docente una actitud crítica y de autoevaluarse en su labor pedagógica. (p. 71)

Por tanto, si bien la participación es importante, el valor real se materializa en la aplicación que el profesorado haga de sus aprendizajes en su contexto de clase y con sus estudiantes. 
Como señalan Bruns y Luque (2014) al referirse a los meta estudios sobre ofertas de capacitación docente:

La pertinencia del contenido, la intensidad y la duración del curso y la calidad de la forma en que se brinda son clave (...) un tema que surge con recurrencia es la necesidad de mejorar las prácticas de los profesores en el aula. (p. 38)

Por lo tanto, el aprendizaje del equipo docente se traducirá en una mejora del aprendizaje de la población estudiantil. El proceso educativo es interactivo y requiere que todas las personas involucradas se comprometan activamente, lo cual implica que el profesorado se responsabilice de su desarrollo profesional realizando los cambios necesarios para mejorar su desempeño docente.

Para la propuesta educativa del PRONIE MEP - FOD la programación y su enseñanza es un tema medular, la apropiación que la población docente sobre el tema es una condición esencial para la implementación de la propuesta. Los resultados apuntan a que el profesorado posee conceptos generales sobre qué es programar, pero no parece suficiente para identificar diferencias en los perfiles en cuanto a prácticas de aprendizaje o desarrollo de destrezas mediante la programación.

A pesar de que las personas docentes identifican dentro de su disciplina la programación como actividad importante en el aprendizaje de la población estudiantil, consideran que requieren más conocimientos para abordarla en el aula. El grupo con mejor desempeño destaca además el aporte de esta disciplina en la resolución de problemas de la vida real, lo que evidencia una mejor comprensión y claridad por parte de este grupo de docentes del papel de la programación en el marco del fortalecimiento y desarrollo de habilidades complejas de pensamiento.

En los perfiles identificados, un resultado significativo es que quienes muestran mayor disposición para la actualización profesional son quienes poseen grados académicos más altos (docentes sobre todo del perfil D). En cuanto al grupo A, son quienes tienen menor experiencia como docentes de IE, se encuentran en la primera etapa de su desarrollo profesional y probablemente han participado poco de ofertas formativas.

A nivel internacional, se ha caracterizado al personal docente que incluye tecnología en educación, como es el caso de los Estándares de la Sociedad Internacional de Tecnologías en Educación (ISTE por sus siglas en inglés) para docentes de Ciencias computacionales

(ISTE, 2011). Esta propuesta describe los conocimientos y prácticas del profesorado de forma similar a la caracterización del personal docente de Informática Educativa del PRONIE MEP-FOD. En otras palabras, como profesionales con el bagaje necesario para desarrollar comprensión, habilidades en alfabetización digital, programación o pensamiento computacional, resolución de problemas y manipulación de datos en el estudiantado.

En América Latina, por ejemplo, Colombia con el Sistema Nacional de Informática Educativa (Galvis, Venegas y Sierra, 2014) o Brasil con el Programa Nacional de Informática Educativa (PRONINFE) (Bianconcini, 2014), desarrollaron iniciativas para incluir las TIC en los ambientes educativos, con un programa de asignatura formal similar al costarricense; no obstante los programas como tales se han transformado en propuestas educativas que incluyen las tecnologías en la educación en diversas modalidades y no estrictamente como una asignatura formal. Por tanto en la actualidad, han desarrollado estándares o competencias para el equipo docente en general que aprovecha las TIC en el ámbito educativo, por citar algunos trabajos: Competencias TIC para el desarrollo profesional docente del Ministerio de Educación nacional de Colombia (2013), competencias TIC para la profesión docente del Ministerio de Educación de Chile, Enlaces (2011). 
En relación con lo anterior, una investigación realizada por la UNESCO (Kalaš et al., 2012) indagó sobre el rol de las TIC en educación primaria, incluyendo nueve centros educativos de Hungría, Jordania, México, Federación de Rusia, Singapur, Eslovaquia, Sudáfrica, Reino Unido y Estados Unidos, dio a conocer las características clave en el rol del profesorado que aproveche las TIC en educación, las cuales coinciden con los desempeños deseados en el personal docente de Informática Educativa costarricense. Por ejemplo, conciben al docente como una persona profesional que se mantiene en procesos de mejora constante en temas de competencias en TIC, proporciona andamiajes para el aprendizaje del estudiantado, promueve la colaboración de estos por medio del uso de computadoras, orienta la adquisición de conocimientos, monitorea y evalúa los logros y productos de aprendizaje de la población estudiantil (Kalaš et al., 2012).

En síntesis, a partir de los resultados de la presente investigación es posible mencionar a nivel general que aunque la mayoría de la población docente de Informática Educativa costarricense se encuentra en los perfiles con mejores puntajes en las dimensiones estudiadas, todavía existe un grupo importante que requiere incrementar sus conocimientos de la propuesta educativa y participar de ofertas de desarrollo profesional.

Con el fin de lograr transformaciones positivas en las prácticas en el laboratorio por medio del aprovechamiento educativo de las tecnologías, es necesario que el personal docente tenga claridad sobre los propósitos educativos por alcanzar, cuál tecnología resulta útil para esos propósitos y cómo usarla, en otras palabras qué tipo de actividad proponer al estudiantado y bajo qué reglas de juego (Zúñiga, 2015). Este tipo de hallazgos promueven retomar la idea de que los cambios esperados con la integración de la tecnología sólo serán posibles considerando en un primer plano cuáles son las barreras por disminuir y los factores por promover, de la mano con cambios en las actitudes, creencias pedagógicas y prácticas de la población docente (Claro, 2010; Pedró, 2011).

\section{CONCLUSIONES}

Según los resultados obtenidos en la evaluación diagnóstica, se puede concluir que la mayoría del profesorado de Informática Educativa se encuentra en los perfiles con mejores puntajes en las dimensiones indagadas, esto significa que la mayoría de ellos y ellas posee conocimientos adecuados que practica en su labor docente y cuenta con algunas habilidades pedagógicas clave características de buenas prácticas educativas en los laboratorios. Esto representa un panorama positivo para ejecutar acciones de desarrollo profesional más adaptadas a las necesidades de la población docente.

El grupo de perfil D, dispuestos a capacitarse y conocedores de la propuesta educativa, evidencia los niveles más altos de uso de tecnología para enseñar frente a otros perfiles. Esto sugiere que quienes más aprovechan el potencial de la tecnología en los espacios educativos son quienes a su vez tienen prácticas más cercanas a lo esperado en lo que se conoce como "buena docencia en Informática Educativa".

Los hallazgos sugieren la necesidad de prestar atención al personal docente que se agrupa principalmente en el perfil A, poco dispuestos a actualizarse y rezagados en la ejecución de la propuesta, en términos de sus características negativas para abordar una propuesta educativa como la de laboratorios, y de hacer conciencia de su responsabilidad inherente a la labor docente de aprovechar al máximo los recursos que el Estado les facilita.

Los bajos puntajes obtenidos en los conocimientos sobre programación y la disposición hacia su enseñanza en todos los perfiles, señalan la necesidad de apuntar al análisis de este eje en la propuesta educativa de Informática Educativa y en las necesidades de actualización profesional sostenida para este sector docente. 
La identificación de los perfiles constituye un insumo útil para crear estrategias de formación profesional más adaptadas a las características del profesorado. Por ejemplo, promoviendo incentivos entre los que poseen mayor motivación y generando acciones articuladas de trabajo con organismos oficiales del país para trabajar con quienes demuestran indisposición ante la actualización en los temas evaluados.

De gran relevancia la toma de acciones en conjunto con el Ministerio de Educación Pública para aplicar medidas al personal docente que no está cumpliendo las expectativas. Un trabajo conjunto permitiría el mejoramiento de las competencias de esta población y por ende la promoción del logro de los resultados de aprendizaje del estudiantado que es el objetivo último de las propuestas educativas del PRONIE MEP-FOD.

Abrir la discusión sobre la calidad de la formación inicial de las personas docentes de Informática Educativa es imperioso para gestar acciones articuladas con las distintas organizaciones interesadas en el tema, que permitan ejercer regulaciones y sentar las responsabilidades debidas para que la población estudiantil costarricense cuente con personal docente de informática calificado y capaz de desarrollar las propuestas pedagógicas exitosamente.

\section{REFERENCIAS}

Bianconcini, M. (2014). Las políticas TIC en los sistemas educativos de América Latina: Caso Brasil. Fondo de las Naciones Unidas para la Infancia (UNICEF): Programa TIC y Educación Básica.

Bruns, B., \& Luque, J. (2014). Profesores excelentes: Cómo mejorar el aprendizaje en América Latina y el Caribe. Estados Unidos: Grupo del Banco Mundial.

Claro, M. (2010). Impacto de las TIC en los aprendizajes de los estudiantes: Estado del arte. Recuperado de: http://repositorio.cepal.org/bitstream/handle/11362/3781/lcw339.pdf;jsessionid=19B35024A5DE1 A53B79D1F851312F751?sequence $=1$

Fundación Omar Dengo (en prensa). Marco de competencias docentes para el aprovechamiento educativo de las tecnologías digitales. San José: Fundación Omar Dengo.

Fundación Omar Dengo (2016). Estudio de conocimientos, prácticas y disposiciones de los docentes de Informática Educativa 2015 (Informe final). San José: Fundación Omar Dengo.

Galvis, A; Venegas, M. y Sierra, L. (2014). Las políticas TIC en los sistemas educativos de América Latina: Caso Colombia. Fondo de las Naciones Unidas para la Infancia (UNICEF): Programa TIC y Educación Básica.

Instituto de Desarrollo Profesional Uladislao Gámez Solano. (2014) Informe: Detección de necesidades de capacitación del personal docente y administrativo docente en servicio del Ministerio de Educación Pública. Costa Rica: MEP.

International Society for Technology in Education (ISTE) (2011). ISTE Standards. Computer Science Educators. Disponible en: http://www.iste.org/standards/standards/ standards-for-computer-science-educators

Kalaš, I., Bannayan, H. E., Conery, L., Laval, E., Laurillard, D., Lim, C. P., Musgrave, S., Semenov, A., \& Turcsányi-Szabó, M. (2012). ICT in Primary Education: Analytical survey. Volume 1: Exploring the origins, settings and initiatives. Moscú: IITE, UNESCO.

Lentini, V. (2014). Perfil sociodemográfico de los docentes en Costa Rica con base en información de los censos 2000 y 2011. Ponencia preparada para el Quinto Informe Estado de la Educación. San José: Programa Estado de la Nación.

Ministerio de Educación nacional de Colombia (2013). Competencias TIC para el desarrollo profesional docente. Disponible en: http://www.colombiaaprende.edu.co/ html/micrositios/1752/articles-318264_recurso_tic.pdf 
Ministerio de Educación de Chile, Enlaces (2011). Competencias TIC para la profesión docente. Disponible en: http://www.enlaces.cl/libros/docentes/index.html

Muñoz, L., Brenes, M., Bujanda, M., Mora, M., Núñez, O. y Zúñiga, M. (2014) Las políticas TIC en los sistemas de América Latina: Caso Costa Rica. País: Fondo de las Naciones unidas para la infancia (UNICEF)

Pedró, F. (2011). Tecnología y escuela: lo que funciona y por qué. España: Fundación Santillana.

Salazar, J. M. (2015). El aprendizaje en los docentes y su integración a la práctica de aula. En Perspectiva educacional: Formación de Profesores, 55-74.

Zúñiga, M. (2015). Usabilidad de las TIC en los procesos de mediación pedagógica. Módulo de capacitación a docentes del proyecto PROEDUCA. Costa Rica: Fundación Omar Dengo

Zúñiga, M., Núñez, O., Brenes, M. y Chacón, D. (2013). La ruta hacia la apropiación de las TIC en los educadores costarricenses. En. Cuarto Informe Estado de la Educación. San José: Programa Estado de la Nación. 